\title{
Classical molecular dynamics simulations of hydrogen plasmas and development of an analytical statistical model for computational validity assessment
}

\author{
M. A. Gigosos,,${ }^{1}{ }^{*}$ D. González-Herrero, ${ }^{1}$ N. Lara, ${ }^{1}$ R. Florido, ${ }^{2}$ A. Calisti, ${ }^{3}$ S. Ferri, ${ }^{3}$ and B. Talin ${ }^{3}$ \\ ${ }^{1}$ Departamento de Física Teórica, Atómica y Óptica, Universidad de Valladolid, 47071 Valladolid, Spain \\ ${ }^{2}$ iUNAT-Departamento de Física, Universidad de Las Palmas de Gran Canaria, 35017 Las Palmas de Gran Canaria, Spain \\ ${ }^{3}$ Aix Marseille Université, CNRS, PIIM, 13397 Marseille, France
}

(Received 16 May 2018; revised manuscript received 10 August 2018; published 17 September 2018)

\begin{abstract}
Classical molecular dynamics simulations of hydrogen plasmas have been performed with an emphasis on the analysis of the equilibration process. The theoretical basis of the simulation model as well as numerically relevant aspects, such as the proper choice and definition of simulation units, are discussed in detail, thus proving a thorough implementation of the computer simulation technique. Because of the lack of experimental data, molecular dynamics simulations are often considered as idealized computational experiments for benchmarking of theoretical models. However, these simulations are certainly challenging and consequently a validation procedure is also demanded. In this work we develop an analytical statistical equilibrium model for computational validity assessment of plasma particle dynamics simulations. Remarkable agreement between model and molecular dynamics results including a classical treatment of the ionization-recombination mechanism is obtained for a wide range of plasma coupling parameter values. Furthermore, the analytical model provides guidance to securely terminate simulation runs once the equilibrium stage has been reached, which in turn gives confidence in the statistics that potentially may be extracted from time histories of simulated physical quantities.
\end{abstract}

DOI: 10.1103/PhysRevE.98.033307

\section{INTRODUCTION}

Computer simulation is the discipline of designing an abstract model to reproduce the dynamics and behavior of an actual physical system, translating the model into a computer program, and analyzing the data obtained from the program execution. With the increase of computational power and data storage, computer simulations have proven to be a valuable tool in many different fields in physics because of their ability for solving complex problems just by relying on fundamental first principles and barely using either physical or mathematical approximations. Results from computer simulations are often considered as idealized experiments, where different effects can be artificially switched on and off to assess their potential impact, thus providing deep insight into the underlying physics and a unique testbed for theory validation.

In particular, the use of computer simulations to study the problem of broadening of spectral line shapes in plasmas has a long history, having significantly contributed to the development and improvement of theoretical models [1]. Nowadays, the theory of Stark broadening has matured enough and become one of the most important diagnostic tools for astrophysical and laboratory plasmas. However, some issues remain still open, e.g., line broadening theory has been validated using independent methods of extracting plasma conditions only for low- $Z$ elements at free electron densities below $10^{25} \mathrm{~m}^{-3}$, disagreement between different approaches persist especially in describing the ion motion effects [2-5], and also discrepancies in the line shape calculations have been pointed

*gigosos@coyanza.opt.cie.uva.es out as the major source of uncertainty in the inferred plasma conditions from the analysis of $K$-shell spectra observed in opacity-related experiments [6]. This scenario has stimulated the research on line broadening over the last few years and led to a series of dedicated workshops for detailed comparisons of computational and analytical methods in order to identify sources of discrepancies and set model validity ranges [7-9].

Computer simulations applied to calculations of Starkbroadened line shapes follow a three-step scheme [1]. The first one consists of simulating the plasma particle dynamics, i.e., the motion of electrons, ions, and neutrals as a result of their mutual interactions of electric nature. Particle dynamics simulations (PDS) provide information about the behavior and statistical properties of local electric microfields, which are ultimately responsible for the Stark broadening and shift of line transitions. In the second step, a representative statistical sample of time histories of the local electric microfield is used to numerically integrate the time-dependent Schrödinger's equation of the radiator, i.e., the emitting ion or atom, and compute the dipole autocorrelation function. In the third step, the Fourier transform of the autocorrelation function, i.e., the power spectral density, is computed, which finally leads to the spectral line profile. In this process, the first step is the most challenging one, since the last two rely on the PDS ability to provide a faithful picture of particle motion and an accurate representation of plasma equilibrium states, which is critical to determine the correct statistics of physical quantities. This work provides insight into the physical and numerical requirements needed for performing reliable PDS.

Mainly, two different approaches have been used over time to simulate the plasma particle dynamics. The first one follows the independent particle approximation (IPA) [2,3,10-16], 
i.e., particle interactions are neglected and they all move following straight-path trajectories. When computing time histories of relevant physical quantities a Debye screened field is assumed to account for coupling effects. Obviously, IPA validity is limited to weakly coupled plasmas. The second approach relies on molecular dynamics (MD) simulations. Now, interactions among all particles are explicitly included and calculations therefore are quite computationally demanding. On the upside, however, collective behaviors (e.g., ion dynamic effects) emerge in a natural way, the range of validity extends to strongly coupled plasmas and, with the lack of experimental data, MD results are often considered as a reference to reveal model deficiencies and provide valuable guidance for theory improvement. Classical MD simulations have been applied to the study of diverse statistical properties, particle correlation effects, and in particular to the investigation of plasma electric microfield distributions [17-25]. Although mainly performed in the context of fully ionized two-component plasmas, all this work enabled the study of electric microfield issues beyond the capability of most theoretical methods. Furthermore, full MD simulations have been used in several works to carry out elaborate calculations of Stark-broadened line profiles in hydrogenic plasmas [14,26-28].

Performing a simulation based on MD techniques is not a straightforward task. A thorough analysis and implementation of numerical and computational modeling of the physics involved are required to optimize the computational time and warrant reliable calculations. In particular, MD simulations have to deal with two pathologic problems: (i) the simultaneous simulation of both light and heavy particles and (ii) the requirement for the system to reach a stationary state in order to provide meaningful statistical samples of the relevant physical quantities. As discussed in Sec. II, the first issue demands a careful analysis of all characteristic time and length scales in the system and its consistent implementation into the numerical algorithms to solve the particle dynamics equations. The second issue is the most delicate one. At the beginning, when Coulomb interactions are switched on, the initial distribution of electrons and ions constitutes a plasma out of equilibrium and an exchange of kinetic and potential energies then takes place between particles throughout the simulation volume. Ideally, at the end of the relaxation phase, statistical measurements can provide an equilibrium temperature together with a density of ion-electron pairs (i.e., recombined ions) and populations of free electrons and ions which fully characterize the equilibrated plasma. In between, the plasma state is slowly evolving and quite undefined until it can be considered as stationary. Thus, in this work we develop a method to carefully control the approach to equilibrium allowing one to know when this slow fluctuating evolution can be securely interrupted to get one of the expected sets of particle positions and velocities, i.e., our technique provides a way to achieve a well-defined plasma equilibrium state.

Also, when simulating plasma particle dynamics, several papers [20,28-32] have discussed the difficulty in dealing with the situation in which an electron is trapped by a charged ion, which may restrict the model applicability to the weakcoupling regime. In this context a few works used MD techniques to model the plasma ionization balance, i.e., including the ionization-recombination mechanism [33-35]. In the simulation model described here the ionization-recombination process is explicitly included within a classical framework, so that recombined ions and neutral pairs are actually native constituents of the final ionization balance and equilibrium state. Our model is therefore appropriate for the study of strongly coupled plasmas beyond the fully ionized scenario, which in turn makes it particularly useful for the calculation of Stark-broadened line shapes. Such study will be addressed on a forthcoming publication. Here, we first focus on demonstrating the robustness and internal consistency of the simulation technique. Thus, benchmarking of numerical algorithms and results is shown for hydrogen plasmas [36], although our technique can be indeed applied for modeling of general multicharged plasmas [37]. Within the framework of classical statistics, we developed an analytical model that mimics the idealized picture of a computer-simulated hydrogen plasma and allows one to obtain the corresponding equilibrium state for given conditions. In this regard, when compared with MD simulation results, the statistical model, firstly, provides a way to prove that a unique equilibrium state has been reached at the end of the relaxation phase and, secondly, leads to a practical definition of a classical atom, which in turn enables the proper definition of a criterion to classify the electron population in the plasma into trapped and free ones. With computer simulations considered as reference numerical experiments, our statistical equilibrium model represents a powerful tool to assess the computational validity of MD simulations and the accuracy of employed numerical methods. To the best of our knowledge, this is the first time in which such crossedcomparison is made.

\section{MOLECULAR DYNAMICS SIMULATION MODEL}

This work focuses on classical MD simulations of particle dynamics of hydrogen plasmas. In this framework, the simulation box is a cube of side $L$ containing $n_{p}$ electrons with mass $m_{e}$ and charge $-q$, and $n_{p}$ ions with mass $m_{i}$ and charge $+q$. Boundary periodic conditions are assumed, i.e., when a particle leaves the box at a given velocity and direction, a particle of the same type enters from the opposite side with exactly the same velocity and direction. At this point it is convenient to recall the definition of some global plasma parameters. Thus, for a hydrogen plasma characterized by a free-electron density $N_{e}$ and an equilibrium temperature $T, r_{0}=\left(3 / 4 \pi N_{e}\right)^{1 / 3}$ gives the average electron-electron distance, and the Debye length, $\lambda_{D}=\left(\varepsilon_{0} k T / q^{2} N_{e}\right)^{1 / 2}$, measures the effective range of Coulomb interactions as a result of the plasma constituents coupling. A characteristic plasma time scale is given by $t_{0} \equiv r_{0} / v_{0}$, where $v_{0} \equiv \sqrt{2 k T / m_{e}}$ is the characteristic electron velocity. It is common to introduce the dimensionless coupling parameter $\rho=r_{0} / \lambda_{D} \propto N_{e}^{1 / 6} T^{-1 / 2}$, with $1 / \rho^{3}$ giving the average number of free electrons within Debye's sphere [13]. A frequent alternative definition of the coupling parameter is $\Gamma=\frac{q^{2}}{4 \pi \varepsilon_{0} r_{0}} \frac{1}{2 k T}$, which represents the ratio between typical Coulomb potential energy and particle kinetic energy. Both parameters satisfy $\Gamma=\rho^{2} / 6$. We note that different combinations of $N_{e}$ and $T$ may lead to the same $\rho$ (or $\Gamma$ ) value. Some representative values are as follows: (a) for arc discharge plasmas, with $N_{e} \sim 10^{22} \mathrm{~m}^{-3}$ and $k T \sim$ 


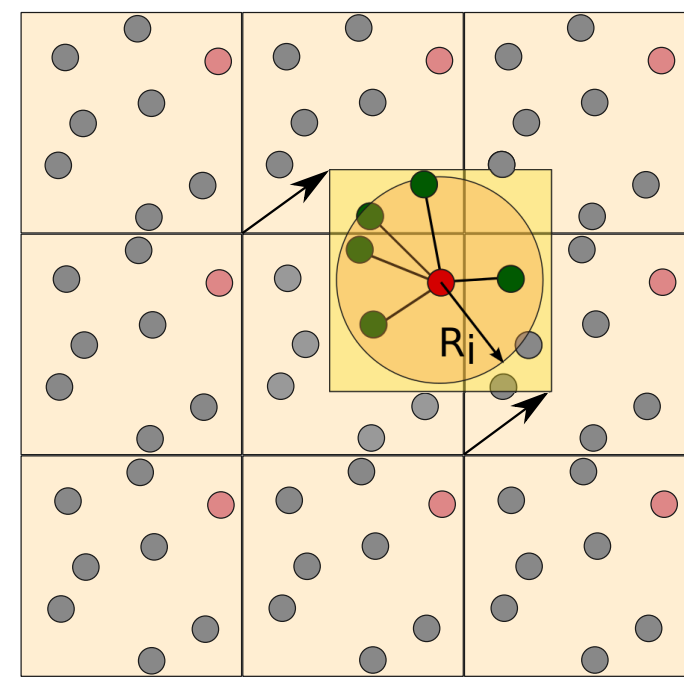

FIG. 1. 2-D representation of the cubic simulation box with periodic boundary conditions. Each particle only interacts with others within a sphere of radius $R_{I}$.

$1 \mathrm{eV}, \rho \sim 0.4\left(\Gamma \sim 3 \times 10^{-2}\right)$; (b) for representative tokamak conditions, $N_{e} \sim 10^{18} \mathrm{~m}^{-3}$ and $k T \sim 1 \mathrm{keV}, \rho \sim 0.003$ $\left(\Gamma \sim 1.5 \times 10^{-6}\right)$; and (c) for an inertial-fusion imploding plasma, with $N_{e} \sim 10^{29} \mathrm{~m}^{-3}$ and $k T \sim 1 \mathrm{keV}, \rho \sim 0.2(\Gamma \sim$ $\left.7 \times 10^{-3}\right)$. In order to keep computational resources and cost within practical limits, each particle is assumed to interact only with charges within a sphere of radius $R_{I}=L / 2$ centered at the particle (see Fig. 1). This sphere of interaction allows one to remove anisotropic effects that naturally arise due to the cubic shape of the whole enclosure. We notice that this assumption does not introduce any additional approximation in the simulation as long as $R_{I} \gg \lambda_{D}$. The latter condition actually sets a lower bound for the number of particles to be used in the simulation, i.e., $n_{p} \gg 1 / \rho^{3}$. In other words, plasma conditions, $N_{e}$ and $T$, determine a minimum number of particles to be included in the simulation. For instance, for $N_{e} \sim 10^{29} \mathrm{~m}^{-3}$ and $k T \sim 1 \mathrm{keV}, n_{p} \gg 125$.

\section{A. Regularized potential}

In order to avoid the collapse of a classical system of ions and electrons interacting through Coulomb forces, the attractive behavior of Coulomb potential should be modified at short distances, thus leading to a finite value at the origin. Such procedure is known as potential regularization and has been extensively discussed in the literature. In summary, two alternatives have been proposed for the choice of a regularized potential. The first one is the use of so-called quantum statistical potentials (QSPs) [38-45], which were devised to take into account short-range quantum effects and avoid divergences in statistical thermodynamics due to Coulomb potential singularity. QSPs were used for the first time in classical MD simulations to investigate hydrogen plasma properties in a strong-coupling regime [17-19]. The second alternative (see Refs. [23,31,33,34]) has a phenomenological origin and was constructed to improve the modeling of ion population kinetics in MD simulations. While QSPs' behavior at short distances typically depends on plasma temperature through the thermal de Broglie wavelength, the latter one is designed to match the corresponding ionization energy at the origin. The impact of using different types of potentials on statistical properties of dense hydrogen plasmas with impurities has been recently studied [24,25]. Neglecting the ionization-recombination mechanism, these works suggest that slow electric microfield distributions are rather insensitive to the potential alternatives and, therefore, such choice would have a small impact on the calculation of Stark-broadened line profiles. The reader interested in these topics is referred to given references for details.

Here we propose a phenomenological ion-electron potential with a quadratic behavior at short distances. A similar model is employed to describe the nuclear interaction in the well-known relativistic self-consistent field Hartree-Fock ATOM package $[46,47]$ and the more recent and widely used FLEXIBLE ATOMIC code [48] for spectroscopic-quality calculations of atomic structure. As shown in Sec. III, this choice has the major advantage of permitting one to develop an analytical plasma equilibrium model, that will be further used to assess the computational validity of our simulation technique. Thus, the ion-electron potential energy $V_{i e}(r)$ is defined as

$$
V_{i e}(r)= \begin{cases}V_{i}\left[\frac{1}{3}\left(\frac{r}{a}\right)^{2}-1\right], & r \leqslant a \\ -\frac{q^{2}}{4 \pi \varepsilon_{0}} \frac{1}{r}, & a<r \leqslant R_{I} \\ 0, & r>R_{I}\end{cases}
$$

with $V_{i}$ denoting the ionization energy, and

$$
a=\frac{3}{2} \frac{q^{2}}{4 \pi \varepsilon_{0}} \frac{1}{V_{i}}
$$

being determined to satisfy continuity and derivability conditions. We note in turn that $a$, which ultimately depends on the ionization energy $V_{i}$, provides an estimate for the characteristic atomic size. From a classical point of view, this potential corresponds to the case of having the ion charge uniformly distributed in the volume of a sphere with radius $a$, which is also permeable to pointlike electrons.

The ion-electron force then results as

$$
\mathbf{F}_{i e}(r)= \begin{cases}-\frac{q^{2}}{4 \pi \varepsilon_{0} a^{3}} \mathbf{r}, & r \leqslant a \\ -\frac{q^{2}}{4 \pi \varepsilon_{0} r^{3}} \mathbf{r}, & a<r \leqslant R_{I} \\ \mathbf{0}, & r>R_{I} .\end{cases}
$$

For $r \leqslant R_{I}$, ion-ion and electron-electron interactions are considered as purely Coulombian, i.e., $V_{i i}(r)=V_{e e}(r)=$ $\frac{q^{2}}{4 \pi \varepsilon_{0} r}$. For $r>R_{I}, V_{i i}(r)=V_{e e}(r)=0$.

\section{B. Ionization-recombination mechanism}

As discussed in Sec. I, several works pointed out the treatment of electron trapping by a charged ion as a delicate issue when performing MD simulations. Here we define a criterion model to deal with such scenario and therefore enable a quantitative control of the ionization-recombination mechanism. Thus, in our simulation model, an electron is considered to be trapped by an ion when (a) their mutual distance is less than the characteristic atomic size, $a$, and (b) the total energy of the pair measured in the center-of-mass reference frame becomes negative. For such calculation, we 
only take into account the potential energy associated to the corresponding electron-ion pair, which is by far the dominant contribution. When this criterion is satisfied, the electron-ion pair is considered as a recombined ion and the electron counts as a bound (trapped) one. Later, due to the interaction with remaining particles, the above-mentioned criterion conditions may not be satisfied anymore, which is interpreted as an ionization, with the electron then returning to the free-electron pool. Within a classical perspective, this dynamic ionizationrecombination process is taken into account throughout the simulation run, thus allowing neutral pairs to be natural constituents of the final equilibrium state. Following this scheme, plasma ionization degree, $\alpha$, can be computed at each time instant.

\section{Particle dynamics and simulation units}

Particle dynamics follows the Newton's second law,

$$
m_{k} \frac{d^{2} \mathbf{r}_{k}}{d t^{2}}=\sum_{\substack{k^{\prime} \\ k \neq k^{\prime}}} \mathbf{F}_{k k^{\prime}}
$$

where $m_{k}$ denotes the mass of the $k$ th particle and $\mathbf{r}_{k}$ gives its position within the cell.

From a numerical perspective, in order to accurately solve the system of motion equations, proper length $r_{p}$ and time $t_{p}$ units have to be chosen. Thus, we can write

$$
\mathbf{r}_{k}=r_{p} \mathbf{x}_{k}, \quad t=t_{p} \tau
$$

where $\mathbf{x}_{k}$ and $\tau$ are dimensionless quantities taking values of the order of 1 in the numerical calculation. In terms of $r_{p}$ and $t_{p}$, electron equations of motion can be rewritten as

$$
\frac{d^{2} \mathbf{x}_{k}}{d \tau^{2}}=\Gamma_{E} \mathbf{F}_{k}^{(e)}
$$

with

$$
\Gamma_{E}=\frac{1}{m_{e}} \frac{q^{2}}{4 \pi \varepsilon_{0}} \frac{t_{p}^{2}}{r_{p}^{3}} .
$$

In Eq. (6), $\mathbf{F}_{k}^{(e)}$ denotes the total force on the $k$ th electron measured in units of $q^{2} / 4 \pi \varepsilon_{0} r_{p}^{2}$, i.e., the simulation force unit. A similar expression is found for ions, in which $m_{e}$ is replaced by $m_{i}$.

The average distance between free electrons, $r_{0}$, might be a tempting choice for the simulation length unit $r_{p}$. However, in the course of the simulation some electrons will be trapped by ions, so that the exact number of free electrons will be known only when achieving the equilibrium state. Therefore, in the simulation, $r_{0}$ is a priori unknown. We then choose for the length unit, $r_{p}$, the average distance between electrons, either free or bound, within the simulation box, i.e.,

$$
\frac{L}{r_{p}}=\left(\frac{4}{3} \pi n_{p}\right)^{1 / 3}
$$

If $n_{e}$ denotes the number of free electrons within the simulation box and $\alpha$ is the ionization degree, then

$$
n_{e}=\alpha n_{p}, \quad \text { with } 0 \leqslant \alpha \leqslant 1 .
$$

According to Eqs. (8) and (9) and the definition of $r_{0}$, we find

$$
\frac{r_{0}}{r_{p}}=\alpha^{-1 / 3}
$$

i.e., the simulation length unit results in a fraction of the freeelectron average distance.

Similarly, the simulation time unit is defined in terms of the plasma characteristic time, $t_{0}$, in such a way that

$$
\frac{t_{0}}{t_{p}}=\beta \text {. }
$$

By substituting Eqs. (10) and (11) into Eq. (7), we find

$$
\Gamma_{E}=\frac{1}{\alpha \beta^{2}} \frac{1}{m_{e}} \frac{q^{2}}{4 \pi \varepsilon_{0}} \frac{t_{0}^{2}}{r_{0}^{3}}=\frac{1}{\alpha \beta^{2}} \Gamma .
$$

Equation (12) suggests to take $\beta=\alpha^{-1 / 2}$, so that the numerical parameter $\Gamma_{E}$ matches the simulated target plasma coupling parameter $\Gamma$, i.e., when launching a simulation, one intends to investigate a plasma at certain target conditions $N_{e}$ and $T$, which leads to the target coupling parameter $\Gamma$. However, as discussed above, the simulation equilibrium state is a priori unknown, so the resulting coupling parameter from the computational experiment, $\Gamma_{\text {expt. }}$, may differ from the originally intended one, $\Gamma$. We note in passing that Eq. (12) sets the way to define the electron charge value in the numerical calculation. Finally, Eq. (11) results as

$$
\frac{t_{0}}{t_{p}}=\alpha^{-1 / 2} \text {. }
$$

This careful choice of simulation units enables our simulation technique to properly deal with the disparate length and timescales that arise in the problem of plasma particle dynamics.

The system of motion equation, Eq. (4), is solved using Verlet's algorithm $[49,50]$ with a certain time step $\Delta \tau$. At each time step, potential, kinetic, and total energy of the system can be easily calculated. Since the system is conservative, total energy must keep constant as time evolves. To numerically satisfy this requirement and find a correct solution of the dynamical equations, a proper $\Delta \tau$ must be used. In this work, $\Delta \tau$ is chosen so that the average of energy numerical fluctuations is equal to zero during the simulation. This is a demanding requirement that typically leads to a short $\Delta \tau$ value and, accordingly, to a high computational cost. Nevertheless, as discussed in Sec. IV B, we benefit from the fact that no numerical heating [51-54] is observed, thus avoiding the use of numerical thermostats [55].

Going through Eqs. (1)-(13), one can see that simulation depends on only two independent physical parameters, i.e., $V_{i}$ and $\Gamma_{E}$, and the number of particles, $n_{p}$. This makes a simulation to exhibit interesting scaling properties. Results obtained in simulation units can be expressed in absolute physical units and an understanding of such unit conversion is very important for the corresponding physical interpretation. For instance, working in simulation units, let us suppose a simulation launched with $V_{i}=4.75\left(\equiv 4.75 \mathcal{E}_{0}\right.$, being $\mathcal{E}_{0}$ the simulation energy unit), $\Gamma_{E}=0.116$, and $n_{p}=255$. Also, suppose that, at equilibrium, the simulation gives $\alpha=0.53$ and we numerically measure a kinetic energy per particle of $\mathcal{E}_{k}=0.50\left(\equiv 0.50 \mathcal{E}_{0}\right)$. Now if we are actually interested 
in simulating a hydrogen plasma, then $V_{i}=13.6 \mathrm{eV}$. This fact sets the simulation energy unit, i.e. $\mathcal{E}_{0}=2.86 \mathrm{eV}$. Since $\mathcal{E}_{k}=\frac{1}{2} m_{e}\left\langle v^{2}\right\rangle=\frac{3}{2} k T$, from the kinetic energy measurement we have the plasma temperature at equilibrium, i.e., $k T=$ $0.95 \mathrm{eV}$. Using Eq. (2), in physical units, the characteristic atomic size results as $a=1.59 \AA$. Considering Eqs. (2) and (7), and taking into account that in simulation units $m_{e}=$ $1, r_{p}=1$, and $t_{p}=1$, in terms of input parameters then we have $a=\frac{3}{2} \frac{\Gamma_{E}}{V_{i}}=0.0366\left(\equiv 0.0366 r_{p}\right)$. Thus, the simulation length unit equivalent is determined, i.e., $r_{p}=43.39 \AA$. Using Eq. (8), one may calculate the size of the simulation box, i.e., $L \approx 443 \AA$. Finally, from Eq. (10) and definition of $r_{0}$, we obtain the plasma electron density at equilibrium, i.e., $N_{e}=1.55 \times 10^{24} \mathrm{~m}^{-3}$.

\section{Setup of initial conditions}

In order to initialize the system of motion equations, initial positions and velocities of all particles in the simulation must be specified. Ion positions are drawn following a uniform distribution within the volume of the simulation box. Around each ion, one electron is randomly placed in a spherical surface of a certain radius. For such configuration, the potential energy is mainly given by the sum of binding energy of ion-electron pairs, since the contribution from the remaining particles is almost negligible. Thus, a proper choice of the ionelectron distance allows one to easily set the initial potential energy to the desired value. Particle velocities are randomly set according to a Maxwellian distribution characterized by a certain temperature and the corresponding mass for each type of particle.

Initial configuration determines the initial potential and kinetic energy and, therefore, the total energy of the system. Also, some of the ion-electron pairs may satisfy the criterion described in Sec. II B to be considered as a recombined ion, so that in general the initial ionization degree does not correspond to the fully ionized state. In other words, the ionization degree is not set by means of any fine-tuning of initial conditions; such initial value is the one that naturally results from the initial configuration.

For $t>0$, the system will evolve undergoing multiple ionizations and recombinations and an exchange between potential and kinetic energy will occur until the system reaches the corresponding equilibrium state and ionization balance. Typically, at $t \sim 0$ a sudden exchange between kinetic and potential energy takes place, which is interpreted as a natural readjustment of the initial configuration. Initially, particle velocities are assigned following a Maxwellian distribution, so that the initial kinetic energy is already well distributed. However, this is not the case of potential energy. At $t \sim 0$ all particles have a potential energy value very similar to the one resulting from the initial draw. This leads to an initial potential energy distribution which resembles a Dirac $\delta$ function and that is certainly far from the one to be reached at equilibrium (see Fig. 14). As a consequence, early in time, such configuration will evolve very quickly. Just a small collective movement of the order of $r_{p}$ is sufficient to produce a significant exchange between kinetic and potential energy. This occurs in a time lapse of the order of $t_{p}$, so definitely the initial energy exchange will be observed as a sudden event compared to the system evolution's typical timescale.

In the entire process, the total energy will remain (within numerical fluctuations) constant. We recall that the generated time histories of physical quantities will be useful for statistical purposes only after equilibrium has been reached. The setup of initial conditions described here permits one to easily manage the balance between the initial potential and kinetic energy, which with the guidance provided by the equilibrium model developed in Sec. III eventually represents a way to speed up the simulation to reach the equilibrium state without any artificial numerical adjustments. In particular, we recall that no thermostat algorithm has been used.

\section{E. Computational resources and details}

Simulations were run in parallel in a computer cluster equipped with a total of 52 graphics processing units (GPUs). All computer programs referred to in this work have been coded from scratch using $\mathrm{C}^{++}$and CUDA ${ }^{\circledR}$. No commercial software or public domain code was used. We actually developed two different codes, one to be run on central processing unit (CPU; sequentially) and the other on GPU (in parallel). This allowed us an easier debugging of our programs, since starting from the same initial conditions, both versions must lead to the same results. Running on CPU was approximately 30 times slower than doing it on GPU, so the CPU version was obviously used only for debugging purposes.

When working on a GPU, the CUDA ${ }^{\circledR}$ programming model distinguishes between threads (the smallest execution unit) and blocks (a group of threads). Also, CUDA ${ }^{\circledR}$ memory hierarchy consists of multiple memory spaces. For instance, each thread has its own private local memory and each block has shared memory visible to all block threads with the same lifetime as the block. In our simulation code, each block deals with one simulation box, i.e., one plasma sample, and each thread within a block is responsible for one single particle. This way all threads run exactly the same piece of code but using different numerical data. Particle locations and interactions between them are saved in the block shared memory, whereas thread local memory stores the velocity of the associated particle. Calculation of interactions is performed in three steps. In the first one, code computes the repulsive force between electrons, the repulsive force between ions in the second step, and the attractive force between ions and electrons in the last one. For calculation of repulsive interactions a do-loop is launched for $i=1, \ldots,\left(n_{p}-1\right) / 2$, with $n_{p}$ always being an odd integer; we recall that $n_{p}$ denotes the number of electrons (and ions) in the simulation. In the $i$ th loop iteration, every $j$ th thread computes the interaction between the $j$ th particle and the one with index $(j+i) \bmod n_{p}$, coded as $(j+i) \% n p$ in $\mathrm{C}^{++}$. Force corresponding to the symmetric configuration is obtained according to Newton's third law and for that reason only $\left(n_{p}-1\right) / 2$ iterations are needed. On every loop iteration, computed force is stored in the block shared memory. This action will never cause a memory conflict because there is not one thread pair working with the same interaction. At the end of every loop iteration, all threads are synchronized, thus preventing any memory conflict during the next iteration. A similar algorithm is used 
for attractive interactions. For these algorithms cache is loaded only once and remains unchanged for the specified number of time steps.

The major limitation of the code comes from the cache size, which restricts the maximum number of particles that can be simulated. All calculations presented here were performed this way, which in turn represents the fastest, well-tested and reliable version of the simulation code. In order to increase the total number of particles, operations must be distributed among different blocks, thus breaking the one-by-one correspondence between plasma samples and blocks. We also developed such a version, which is slower due to required synchronization between different blocks and consequently more prone to cache faults occurrence.

The entire set of calculations (not all of them shown here) and analyses performed to build up the present research spanned over six wall-clock time months. In total, we carried out 1488 independent simulations that took a range of computational times from 1 to 81 days (typically 5 days) depending on the physical conditions of the simulated plasma.

\section{ANALYTICAL STATISTICAL EQUILIBRIUM MODEL}

Computer simulations are often considered as idealized experiments providing a unique testbed for validation of theoretical models. In this regard, an assessment of simulation reliability becomes a critical task that has not always received the attention it deserves. Here we have developed an analytical model to describe the equilibrium state of a hydrogen plasma and thus checking the reliability and validity of the computer simulations. This model does not aim to describe the behavior of a real plasma but to mimic the physical conditions in which simulation takes place and properly describe the corresponding statistics.

Dissipative radiative processes are not taken into account in the simulation, so the ionization balance appears as a result of collisional ionization and recombination processes. In this context, population kinetics is ruled by the well-known Saha equation [56],

$$
\frac{n_{e} n_{i}}{n_{n}}=\frac{Z_{e}(T) Z_{i}(T)}{Z_{n}(T)},
$$

wherein $n_{e}, n_{i}$, and $n_{n}$ are the number of free electrons, ions, and neutral atoms (in the simulation a neutral atom consists of a bound electron-proton pair) in the plasma, respectively, and $Z_{e}(T), Z_{i}(T)$, and $Z_{n}(T)$ are the corresponding classical partition functions at equilibrium temperature $T$.

Particle dynamics is ruled by laws of classical mechanics and accordingly it will show classical statistical properties. Hence, the free-electron partition function is given by

$$
\begin{aligned}
Z_{e}(T) & =\int_{V} d^{3} \mathbf{r} \int d^{3} \mathbf{p} \exp \left(-\frac{p^{2}}{2 m_{e} k T}\right) \\
& =V\left(2 \pi m_{e} k T\right)^{3 / 2} .
\end{aligned}
$$

Similarly, for ions we have

$$
Z_{i}(T)=V\left(2 \pi m_{i} k T\right)^{3 / 2} .
$$

Lastly, the neutral atoms partition function is obtained as

$$
Z_{n}(T)=Z_{n \text { trans }}(T) Z_{n \text { int }}(T)
$$

with

$$
\begin{aligned}
Z_{n \text { trans }}(T) & =\int_{V} d^{3} \mathbf{r} \int d^{3} \mathbf{p} \exp \left(-\frac{p^{2}}{2 m_{n} k T}\right) \\
= & V\left(2 \pi m_{n} k T\right)^{3 / 2} \\
Z_{n \text { int }}(T)= & \int_{\mathcal{E}<0} d^{3} \mathbf{r} \int d^{3} \mathbf{p} \exp \left\{-\frac{1}{k T}\left[\frac{p^{2}}{2 \mu}+V_{i e}(r)\right]\right\} \\
= & \left(\frac{3 \pi k T a^{2}}{V_{i}}\right)^{3 / 2}(2 \pi \mu k T)^{3 / 2} e^{V_{i} / k T} \\
& \times\left\{1-e^{-V_{i} / k T}\left[1+\left(\frac{V_{i}}{k T}\right)+\frac{1}{2}\left(\frac{V_{i}}{k T}\right)^{2}\right]\right\} .
\end{aligned}
$$

Here, $V$ stands for the plasma volume, $m_{n}=m_{i}+m_{e}$, and $\mu=m_{i} m_{e} / m_{n}$ is the ion-electron reduced mass. $Z_{n \text { trans }}(T)$ denotes the translational partition function, i.e., resulting from the movement (translation) of the center of mass, and $Z_{n \text { int }}(T)$ is the internal partition function, which accounts for internal degrees of freedom. In Eq. (19) the integration domain is limited to the phase-space region satisfiying

$$
\mathcal{E}(r, p)=\frac{p^{2}}{2 \mu}+V_{i e}(r)<0
$$

as happens in a bound system. We also note that the choice of the quadratic behavior for $V_{i e}(r)$ at short distances, Eq. (1), leads to an analytical solution for the coordinates integral in Eq. (19).

Thus, our classical Saha equation results as

$$
\frac{N_{e} N_{i}}{N_{n}}=\left(\frac{V_{i}}{k T} \frac{1}{3 \pi a^{2}}\right)^{3 / 2} \frac{e^{-V_{i} / k T}}{1-e^{-V_{i} / k T}\left[1+\left(\frac{V_{i}}{k T}\right)+\frac{1}{2}\left(\frac{V_{i}}{k T}\right)^{2}\right]},
$$

with $N_{x}=n_{x} / V$ and $x \equiv e, i, n$.

With $N_{p}=N_{i}+N_{n}$, in terms of the ionization degree, $\alpha$, we have $N_{e}=N_{i}=\alpha N_{p}$ and $N_{n}=(1-\alpha) N_{p}$. Then, Eq. (21) becomes

$$
\frac{\alpha^{2}}{1-\alpha}=K(T)
$$

being

$$
\begin{aligned}
K(T)= & \frac{4}{9 \sqrt{3 \pi}}\left(\frac{r_{p}}{a}\right)^{3}\left(\frac{V_{i}}{k T}\right)^{3 / 2} \\
& \times \frac{\mathrm{e}^{-V_{i} / k T}}{1-e^{-V_{i} / k T}\left[1+\left(\frac{V_{i}}{k T}\right)+\frac{1}{2}\left(\frac{V_{i}}{k T}\right)^{2}\right]},
\end{aligned}
$$

where $N_{p}=1 /\left(\frac{4}{3} \pi r_{p}^{3}\right)$. As seen from Eq. (23), plasma ionization balance does not depend on ion mass. Thus, we finally obtain a compact analytical formula for the plasma ionization degree,

$$
\alpha=\frac{K(T)}{2}\left[\sqrt{1+\frac{4}{K(T)}}-1\right]
$$


TABLE I. Summary of the entire set of calculations performed to build up the present work. Specifications of the electron-positron and electron-proton simulated plasmas are given. These include (a) the numerical coupling parameter, $\Gamma_{E} ;$ (b) the ionization energy, $V_{i} / \mathcal{E}_{0}$; (c) the number of electrons, $n_{p}$, included in the simulation, which equals the number of positrons or protons; the ranges of initial (d) kinetic and (e) potential energy [examples of specific initial values are indicated as open circles (green) associated to $t=0$ in Fig. 10]; (f) the range of time step values used to integrate the motion equations, $\Delta t / t_{p} ;(\mathrm{g})$ the range of values for the number of time steps reached in the simulation; and (h) the number of simulation runs associated to the specified input parameter values (indicated as $c \times g$, where $c$ is the number of cases and $g$ is the number of plasma samples sharing the same initial physical conditions).

\begin{tabular}{|c|c|c|c|c|c|c|c|}
\hline \multicolumn{8}{|c|}{ Type of plasma: Electron-positron } \\
\hline \multicolumn{3}{|c|}{ Input parameters } & \multicolumn{2}{|c|}{ Initial conditions } & \multirow{2}{*}{$\frac{\text { Time step }}{\Delta t / 10^{-4} t_{p}}$} & \multirow{2}{*}{$\frac{\text { No. steps } / 10^{6}}{t_{\text {total }} / 10^{6} \Delta t}$} & \multirow{2}{*}{$\begin{array}{l}\text { No. simulation } \\
\text { runs }\end{array}$} \\
\hline$\Gamma_{E}$ & $V_{i} / \mathcal{E}_{0}$ & $n_{p}$ & $\mathcal{E}_{k} / \mathcal{E}_{0}$ range & $\mathcal{E}_{p} / \mathcal{E}_{0}$ range & & & \\
\hline 0.1161 & 3.00 & 255 & {$[0.00,3.00]$} & {$[-1.25,-0.25]$} & 4 & {$[140,300]$} & $18 \times 8$ \\
\hline 0.1161 & 4.75 & 255 & {$[0.00,3.00]$} & {$[-2.25,-0.25]$} & {$[1,5]$} & {$[36,300]$} & $53 \times 8$ \\
\hline 0.1161 & 5.50 & 255 & {$[0.00,3.00]$} & {$[-2.70,-0.25]$} & {$[2,4]$} & {$[133,300]$} & $20 \times 8$ \\
\hline 0.1161 & 6.80 & 255 & {$[0.00,3.00]$} & {$[-3.25,-0.25]$} & {$[1,5]$} & {$[67,237]$} & $33 \times 8$ \\
\hline 0.0417 & 4.75 & 425 & {$[0.00,3.00]$} & {$[-2.00,-0.25]$} & 2 & {$[15,1445]$} & $15 \times 8$ \\
\hline 0.0417 & 6.80 & 425 & {$[0.00,3.00]$} & {$[-3.25,-0.25]$} & 5 & {$[487,1309]$} & $33 \times 8$ \\
\hline
\end{tabular}

Type of plasma: Electron-proton

\begin{tabular}{|c|c|c|c|c|c|c|c|}
\hline \multicolumn{3}{|c|}{ Input parameters } & \multicolumn{2}{|c|}{ Initial conditions } & \multirow{2}{*}{$\frac{\text { Time step }}{\Delta t / 10^{-4} t_{p}}$} & \multirow{2}{*}{$\frac{\text { No. steps } / 10^{6}}{t_{\text {total }} / 10^{6} \Delta t}$} & \multirow{2}{*}{$\begin{array}{l}\text { No. simulation } \\
\text { runs }\end{array}$} \\
\hline$\Gamma_{E}$ & $V_{i} / \mathcal{E}_{0}$ & $\overline{n_{p}}$ & $\mathcal{E}_{k} / \mathcal{E}_{0}$ range & $\mathcal{E}_{p} / \mathcal{E}_{0}$ range & & & \\
\hline 0.1161 & 4.75 & 255 & {$[0.00,3.00]$} & {$[-2.00,-0.25]$} & {$[0.5,5]$} & {$[40,1886]$} & $14 \times 8$ \\
\hline
\end{tabular}

As shown in detail in Sec. IV B, the last equation will be very useful to analyze the results provided by the numerical simulations.

\section{Equilibrium curve}

Besides providing the plasma ionization degree, the analytical model allows us to build an equilibrium curve, i.e., a potential energy vs kinetic energy plot that shows how the total energy distributes on each equilibrium state. by

In this context, the average kinetic energy is simply given

$$
\mathcal{E}_{k}=\frac{3}{2} k T
$$

and the average potential energy of free particles is considered to be equal to zero. The potential energy of an ion-electron pair in a neutral atom is given by its binding energy, since this is by far the most important contribution and the ones from remaining particles can be neglected. Hence, the potential energy per bound particle is written as

$$
\mathcal{E}_{p b}=\frac{1}{2}\left(-V_{i}+\frac{3}{2} k T\right),
$$

as corresponds to a parabolic potential. The $\frac{1}{2}$ common factor distributes the binding energy between the proton and the electron in the pair. The potential energy per particle is given by

$$
\mathcal{E}_{p}=\frac{1-\alpha}{2}\left(-V_{i}+\frac{3}{2} k T\right)
$$

\section{RESULTS}

The investigation addressed in this work relies on a significant number of MD simulations of both electron-positron and electron-proton plasmas. Comprehensive studies to check the consistency of the simulation code and physical behavior with respect to simulation parameters like the potential-energy well depth or the coupling parameter were performed. For the sake of clarity, the entire set of calculations is summarized in Table I, with indications of input parameter values and other simulation details, such as the chosen time step and kinetic- and potential-energy initial conditions. Obviously, there is no point in showing all the collected calculations here. Representative results have been properly chosen according to the aims of this work: the study of the equilibration process and the computational validity assessment of our simulation model. These topics and results are discussed in the following Secs. IV A and IV B.

\section{A. Equilibration process}

In a MD simulation the statistical sampling of relevant physical quantities and processes is only meaningful once particle dynamics becomes stationary and the system thus reaches the equilibrium state. This means that a MD calculation has to go through an initial equilibration or relaxation stage, which is by itself useless to get relevant physical information, but in turn necessary to drive the system to the stationary stage from which the statistical sampling can be safely performed. From a computational point of view, the time needed to reach the equilibrium state in a simulation run is substantial. Typically, for MD simulations of positron-electron plasmas, equilibration time easily hits a few thousands of simulation time units, i.e., $\sim 10^{3} t_{p}$, which is in agreement with the results obtained in Ref. [27]. With the choice of an integration time step of the order of $10^{-4} t_{p}$ for solving the system of motion equations, reaching the stationary stage therefore requires several millions of time steps. In this regard, caution must be taken to not prematurely 


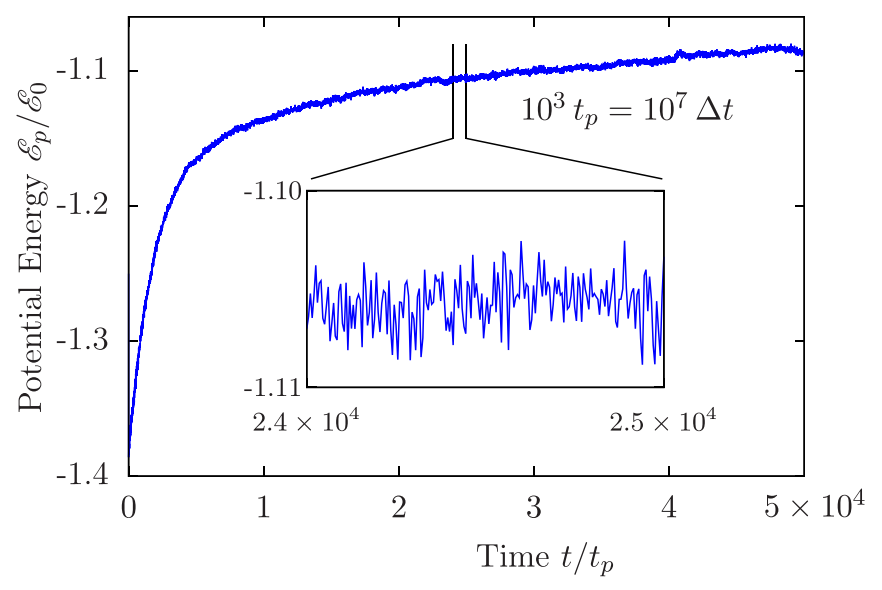

FIG. 2. Time evolution of potential energy per particle in a simulation of an electron-proton plasma $\left(m_{p}=1836 m_{e}\right)$. Throughout the thermalization process, the inspection of a time interval including tens of millions of steps may result in being too short to securely identify that equilibrium has been reached.

terminate simulation runs, which might lead to an inaccurate statistics of physical quantities.

Equilibration time further increases with mass difference between plasma constituents (electrons and heavier ions, for instance), since very different timescales appear than involved in motion equations. Figure 2 illustrates the delicate and slow process of plasma equilibration. In this case a proton-electron plasma (i.e., hydrogen plasma) has been simulated. The figure shows the time evolution of potential energy per particle. From the inset plot it is clear that when looking at a time interval including only tens of millions of time steps the change in energy appears hidden by numerical fluctuations. This may lead to wrongly thinking that the equilibrium has been achieved and thus prematurely terminate the simulation run. In the example, the true equilibrium state is still far from being reached. In this regard, it is certainly helpful to have a model such as the one described in Sec. III to provide guidance about the equilibrium point $\left(\mathcal{E}_{k}^{\mathrm{eq}}, \mathcal{E}_{p}^{\mathrm{eq}}\right)$ and undoubtedly identify the thermalization of the simulated plasma. We note in passing that, in this work, thermalization (i.e., equilibrium state) is considered to occur when statistical distributions of all physical quantities become stationary.

The equilibration process is much faster in a positronelectron plasma than in a proton-electron case. For comparison, time evolution of kinetic and potential energy per particle, and plasma ionization degree are shown in Figs. 3-5, respectively. While ions take more time to thermalize, electron kinetic energy rapidly reaches the stationary state even for a hydrogen plasma. A slower path to equilibrium is observed for the case of potential energy, i.e., particle spatial distribution takes longer to achieve an equilibrium configuration. This is particularly important because our simulations are ultimately aimed to study and characterize the local electric field properties, and obviously the corresponding dynamics and statistics are ruled by particle spatial arrangement.

Keeping in mind the criterion adopted in the simulation to model the ionization-recombination mechanism (described in Sec. II B), the plasma ionization degree can be computed

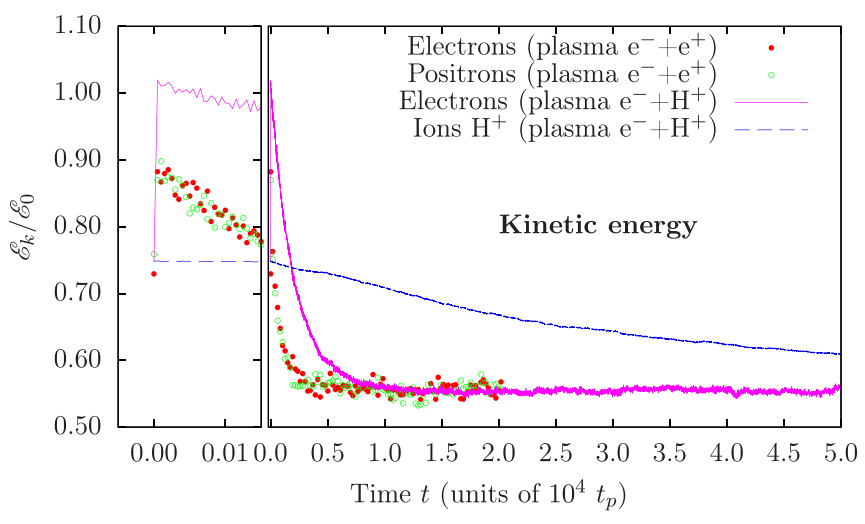

FIG. 3. Comparison of the equilibration process between positron-electron and proton-electron plasmas. Here we plot the time history of the kinetic energy per particle. The characteristic sudden evolution early in time from the initial configuration has been zoomed in in the left box.

at each time instant throughout the system evolution. An example is shown in Fig. 5. Compared to kinetic and potential energy, the ionization degree shows the slowest approach to equilibrium. This is because, as suggested before, from a classical perspective the ionization balance equilibration process is basically ruled by the less-frequent three-body processes. Thus, even when a small change in the kinetic energy of a given electron or in the potential energy associated to a positron-electron (or ion-electron) pair has a negligible influence on the corresponding average values, such a small change may determine the difference for an electron to be considered either as a bound or a free one, which indeed has a greater impact on the calculation of the ionization degree. Stationarity assessment of this parameter is crucial, since it determines the free-electron density value, which is a key quantity for the statistical analysis of the local electric field.

As Eqs. (23), (24), and (27) suggest, neither energy distribution among particles nor partition between kinetic and potential energy depend on particle mass. We have both numerically confirmed this fact and taken advantage of it to speed up the simulation of hydrogen plasmas. We launch the calculations using positrons and once the equilibirum

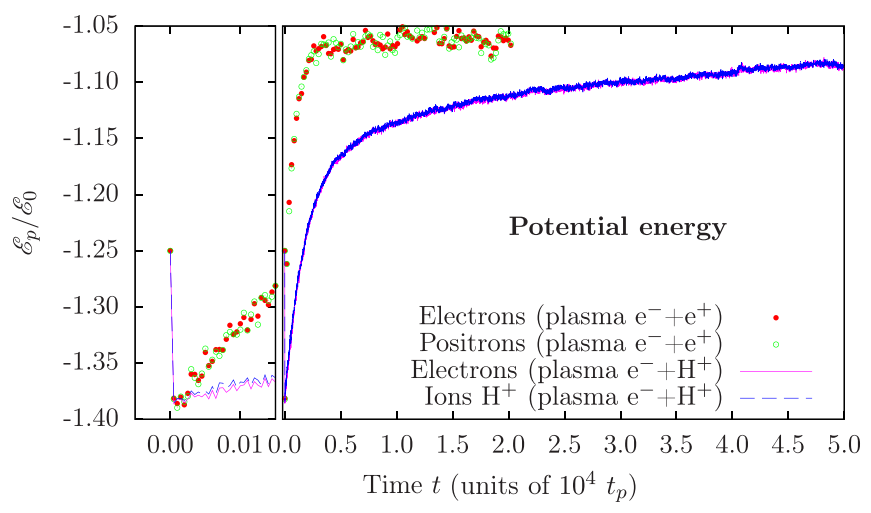

FIG. 4. Time history of potential energy per particle for the same cases shown in Fig. 3. 


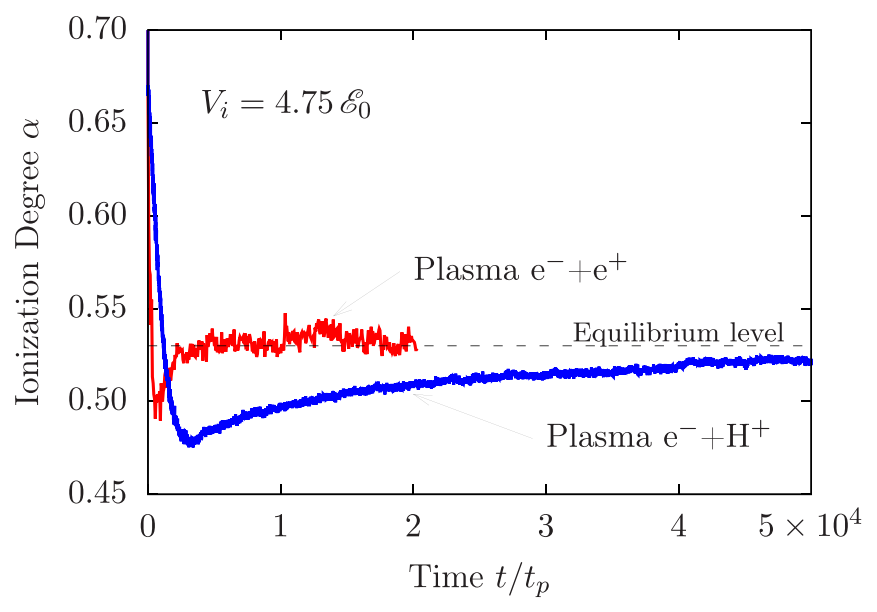

FIG. 5. Time history of plasma ionization degree for the same cases shown in Figs. 3 and 4.

is reached, positron mass is replaced by proton mass and velocity moduli are modified accordingly to keep the right kinetic energy values. Thenceforth, taking the particle spatial distribution at the switch time, the simulation proceeds with updated masses and velocities.

We illustrate this point in Figs. 6-8. Figure 6 shows time histories of kinetic, potential, and total energy per particle. The evolution of the plasma ionization degree is plotted in Fig. 7. When going through the positron-proton switch time, we did not observe any appreciable difference in either average energy values or plasma ionization degree, which show the characteristic steady behavior at equilibrium. Also, with the only expected exception of the ion velocity distribution, it is seen that statistical distributions of the system do not change with the positrons-by-protons (ions) replacement. An illustration is given in Fig. 8. For the case of positrons, the potential energy statistical distribution shown in the figure actually

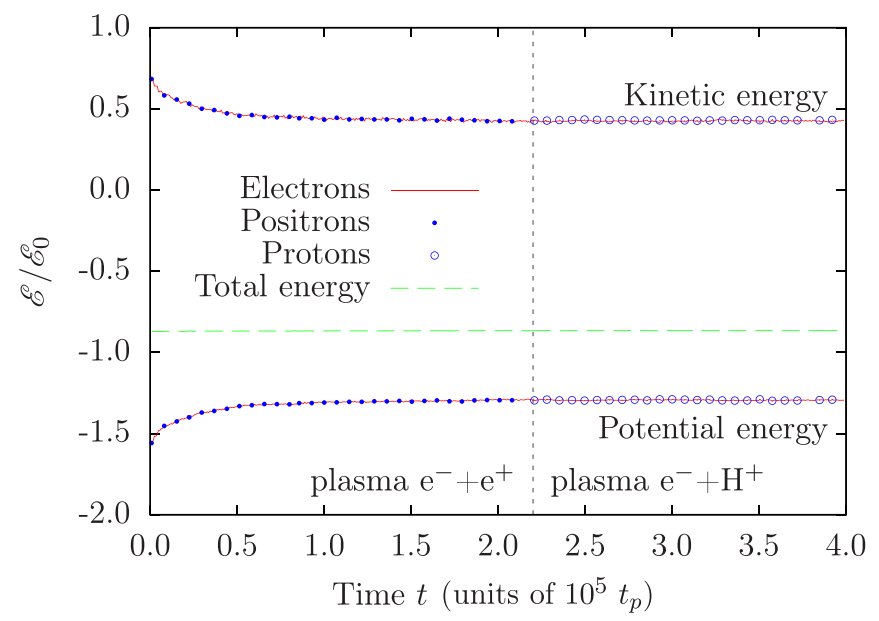

FIG. 6. Time histories of kinetic, potential, and total energy per particle for a simulation that begins as an electron-positron plasma and converts to a hydrogen plasma. The positron-by-proton replacement occurs at $t_{c}=2.2 \times 10^{5} t_{p}$. The plot illustrates the technique to speed up the process for achieving the equilibrium in a hydrogen plasma.

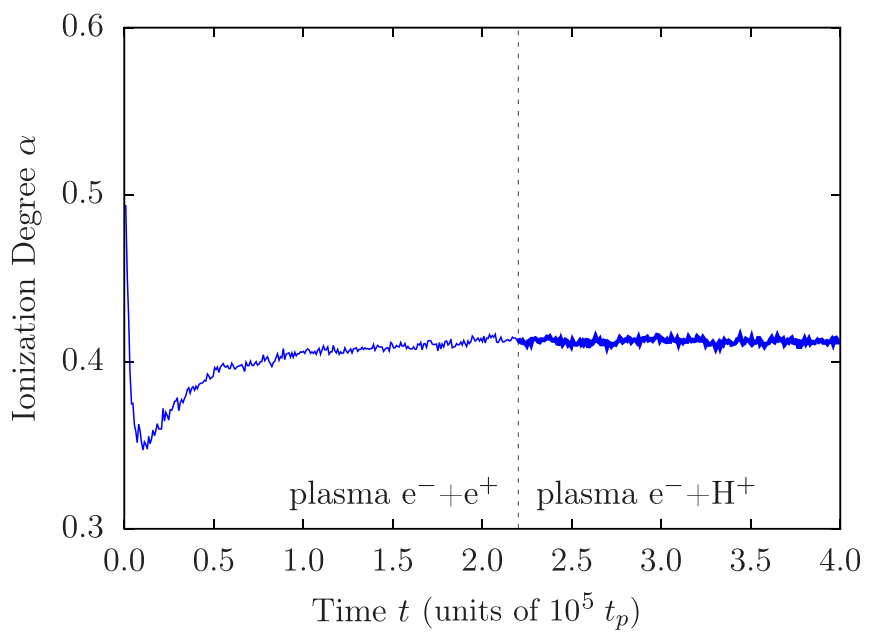

FIG. 7. Time history of plasma ionization degree for the same case shown in Fig. 6.

represents the average result over eight simulation runs with the same plasma macroscopical physical conditions sampled at the time $t_{c}$, i.e., right before the replacement. The distribution corresponding to hydrogen ions has been obtained using data from the same eight independent simulations but further sampling each simulation every 1000 time units throughout a $2.64 \times 10^{5}$-units-long time interval at equilibrium conditions (i.e., the results shown in the figure actually sum up the data from 2112 different plasma configurations taken from simulation time histories within the stationary stage). We also note that the duration of the referred interval after switch time is equivalent to $\sim 6000$ times the proton characteristic time, which is long enough for each proton to go $\sim 700$ times across the simulation box. As seen, good consistency between distributions before and after updating the mass and velocity of positively charged particles was obtained.

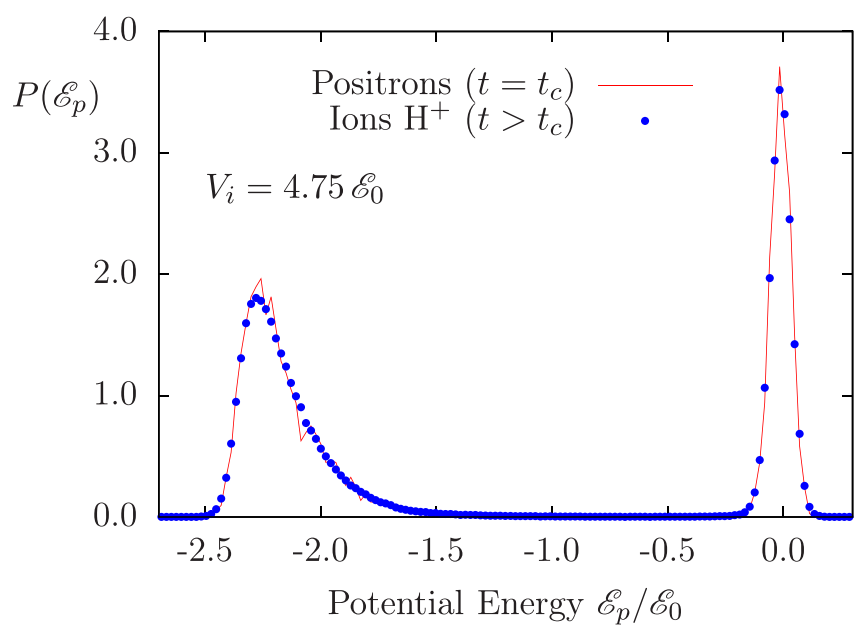

FIG. 8. Statistical distribution of potential energy per particle. Results are shown for (a) positrons (in an electron-positron plasma) at the time $t_{c}$ of positron-by-proton replacement and (b) protons (electron-proton plasma) after the switch time (see the text for details). 


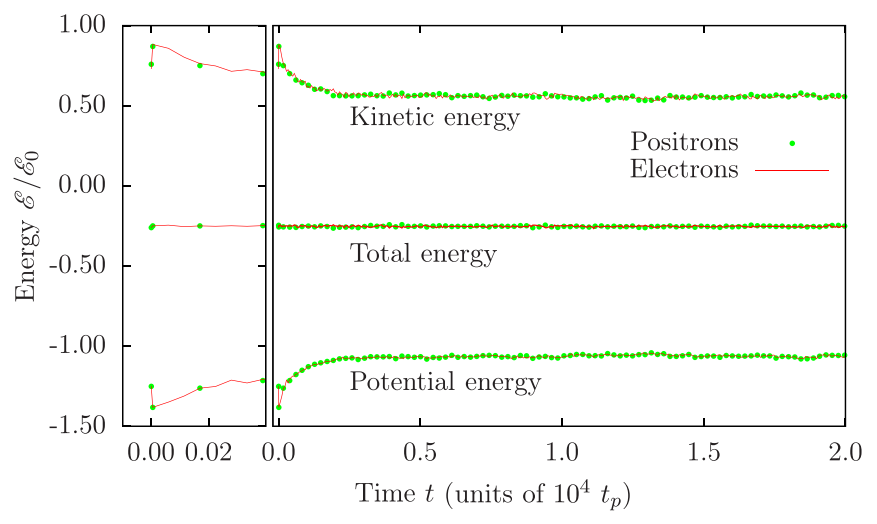

FIG. 9. Illustration of kinetic, potential, and total energy time histories in a simulation. For the case shown, the average kinetic and potential energies in the initial configuration were $\mathcal{E}_{k}=0.74 \mathcal{E}_{0}$ and $\mathcal{E}_{p}=-1.25 \mathcal{E}_{0}$, respectively; and $V_{i}=4.75 \mathcal{E}_{0}$. Results represent the average over eight simulations launched with the same initial average kinetic and potential energies. On the left side, simulation earliest stage has been zoomed in. On the right side, the entire time history is shown. For the sake of clarity, only 1 every 10 calculation time steps is displayed in the electron time histories and only 1 every 40 in the case of positrons.

\section{B. Comparison with the equilibrium model and computational validity assessment}

Our goal in this section is the computational validity assessment of simulation results by comparison with the analytical theoretical model described in Sec. III. Results shown here were obtained from a considerable number of positron-electron plasma simulations. As shown in Sec. IV A, the system properties and configuration at equilibrium do not depend on particle masses, so we take advantage of this fact and use positrons with the only purpose to speed up the simulations and reduce the computational time to achieve the equilibrium. For each simulation, the positions and velocities of 255 (or 425) positrons and electrons were drawn according to the procedure described in Sec. II D. In order to obtain more accurate statistical distributions and average values, for each pair $\left(\mathcal{E}_{k}, \mathcal{E}_{p}\right)$ of kinetic and potential energies, we performed from 8 to 64 completely independent simulations, i.e., initial positions and velocities were different, but yielding the same average potential and kinetic energy values per particle.

As discussed in Sec. II, numerical algorithms employed in our simulation model are robust enough, so that throughout the evolution of simulated plasma no external control procedure of total energy value was needed. The time step for integration of motion equations was chosen between $5 \times 10^{-5}$ and $5 \times 10^{-4}$ depending on the case, i.e., time step value is taken lower the greater the average kinetic energy. As a rule of thumb, in a time step a plasma particle travels a distance of the order of $10^{-4}$ times the characteristic interparticle distance. No numerical heating was observed.

For illustration of the equilibration process in the simulation runs shown in this section, in Fig. 9 we plot the evolution of kinetic, potential, and total energy per particle for a given simulation case. The results have been obtained by averaging eight plasma simulations launched with the same initial kinetic and potential energies. Typically, at the

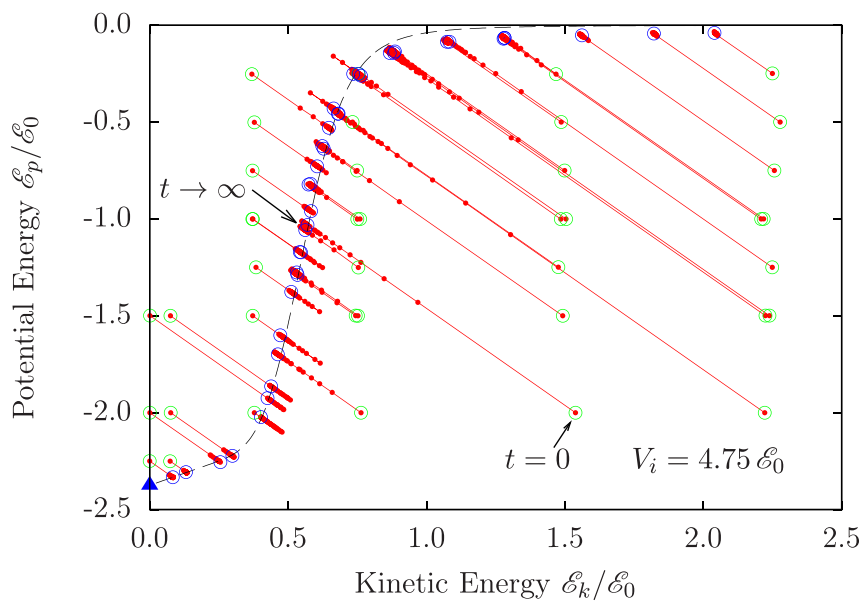

FIG. 10. Comparison between the equilibrium curve predicted by the analytical model (dashed line) and the one obtained from numerical simulation runs. Path to equilibrium for each simulation run (solid circles) follows a $\mathcal{E}_{k}+\mathcal{E}_{p}=$ cte trajectory. Starting, i.e., initial configuration, and final points of simulation trajectories are indicated by open circles (green and blue, respectively). The triangle dot indicates the minimum energy state of the system, i.e., the case of a static distribution of ions and bound electrons. Simulation input parameters were $V_{i}=4.75 \mathcal{E}_{0}$ and $\Gamma_{E}=0.116$.

earliest stage of the simulation, a sudden change in both particle positions and velocities happens as a consequence of a kinetic and potential energy exchange. Then, a slower evolution toward the equilibrium is observed. In the case shown, a transfer from kinetic to potential energy occurred, although the opposite might happen in other cases. As seen, total energy is conserved throughout the entire simulation and only the characteristic numerical fluctuations are observed.

In order to illustrate the validity assessment of our MD code, comparisons between the analytical equilibrium model and the simulation results are displayed in Figs. 10, 11, and 13. In particular, Fig. 10 shows the equilibrium curve as predicted

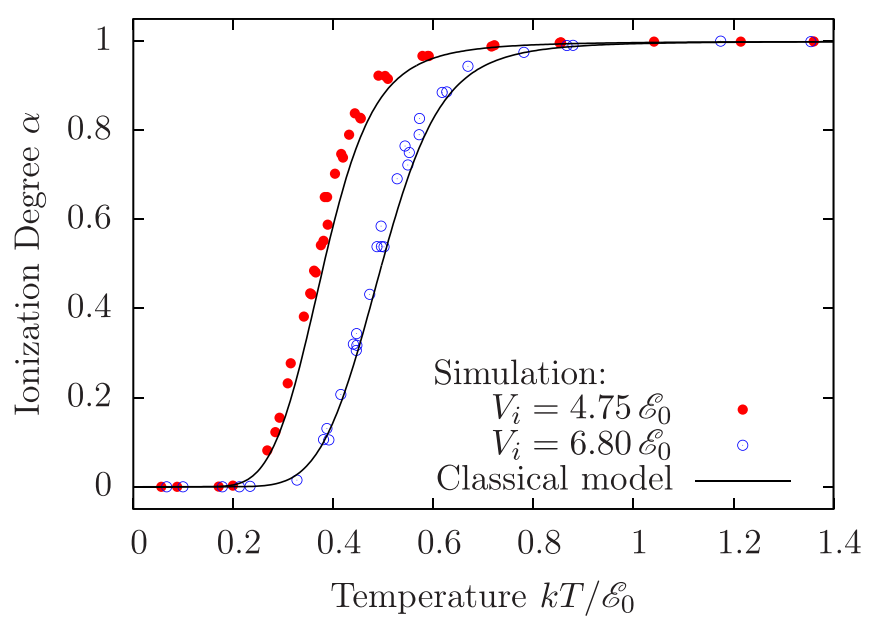

FIG. 11. Ionization degree as a function of temperature at equilibrium. Comparisons between the analytical model and simulations are shown for two cases, i.e., $V_{i}=4.75 \mathcal{E}_{0}$ and $V_{i}=6.80 \mathcal{E}_{0}$ (with $\left.\Gamma_{E}=0.116\right)$. 
by the model according to Eqs. (25)-(27). Paths to equilibrium in such $\mathcal{E}_{p}: \mathcal{E}_{k}$ plane are also plotted for a collection of 40 different simulation cases. Potential and kinetic energy values of initial configurations span over an interval broad enough to survey the equilibrium curve in a wide temperature range. For each case, the plasma approaches the equilibrium by means of an exchange between kinetic and potential energy, and always following a $\mathcal{E}_{k}+\mathcal{E}_{p}=$ cte trajectory. When simulation starts at a point below the model equilibrium curve, the simulated plasma cools down and the average potential energy increases. Oppositely, there is a transfer from potential to kinetic energy when the initial configuration lies above the equilibrium curve. A remarkable agreement between the equilibrium curve obtained from the analytical model and the one defined by simulation runs is observed.

Figure 11 shows the ionization degree as a function of temperature at equilibrium. Two different simulation groups are plotted, for $V_{i}=4.75 \mathcal{E}_{0}$ and $V_{i}=6.80 \mathcal{E}_{0}$, respectively. Overall, when comparing with model predictions, i.e., Eq. (23), a good agreement is observed, with simulated ionization degree slightly overestimating model results. Differences come from the way in which electrons are classified as bound or free in either the model or the numerical simulation. In the theoretical model, a chemical picture is inherently used, and plasma constituents are viewed as atoms, ions, and free electrons. Hence, bound and free electrons are clearly distinguished. MD simulations, however, naturally develop within a physical picture, where interactions among particles are treated on an equal many-body footing. The frontier between bound- and free-electron concepts is not so well defined and a criterion, like the one described above, must be specified to perform such classification and determine the ionization degree. From a comparison like the one shown in Fig. 11, a numerical criterion to match with the results that arise from the chemical picture implicitly assumed in the model could be extracted. However, as discussed below, this would require a deeper investigation on the existence and treatment of collectivized electrons in the plasma, which is beyond the scope of this work.

Once the equilibrium is reached, corresponding temperature, ionization degree, and free-electron density values are obtained, and therefore the experimental plasma coupling parameter (i.e., as a result of the computational experiment) can be determined. The simulation results shown in Figs. 10 and 11, using a fixed numerical input parameter $\Gamma_{E}=0.116$, lead to experimental coupling parameter values in the range $0 \lesssim \Gamma_{\text {expt. }} \lesssim 0.13$ (see Fig. 12), thus spanning from weakly to moderately coupled plasmas. We here recall the physical meaning and difference between dimensionless parameters $\Gamma_{E}$ and $\Gamma$, as described above in Sec. II C. Numerical parameter $\Gamma_{E}$ controls the strength of particle interactions [see Eq. (6), which is written in simulation units]. In other words, $\Gamma_{E}$ defines the magnitude of electron charge in the simulation. Coupling parameter $\Gamma$ instead is determined by the ratio between the typical free-electron interaction energy and the corresponding kinetic energy, i.e., it gives a relation between free-electron density and electron temperature, and therefore accounts for the system coupling degree. When a simulation is launched, a certain amount of energy is delivered to the system by means of draw-resulting initial conditions.

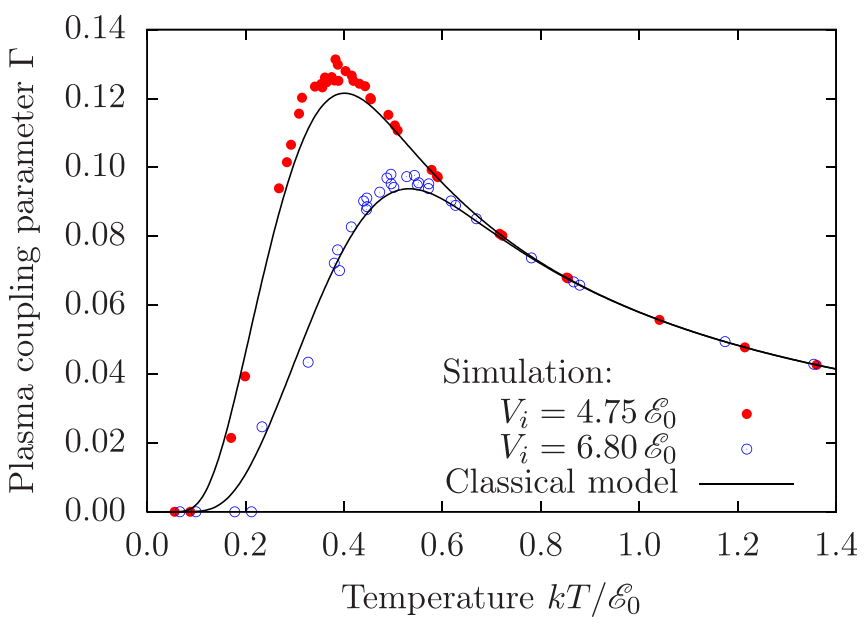

FIG. 12. Plasma coupling parameter as a function of temperature at equilibrium. Comparisons between the analytical model and simulations are shown for two cases, i.e., $V_{i}=4.75 \mathcal{E}_{0}$ and $V_{i}=6.80 \mathcal{E}_{0}$ (with $\Gamma_{E}=0.116$ ).

The system consistently evolves according to interaction strength fixed by $\Gamma_{E}$ and energy is redistributed to eventually reach an equilibrium state and ionization balance with well-defined values for kinetic and potential energy. At such equilibrium state we can measure the resulting free-electron density and temperature, and therefore obtain $\Gamma_{\text {expt. }}$. As observed in Fig. 12, in the low-temperature limit, plasma mainly consists of noninteracting neutral pairs, and consequently coupling is weak. As the temperature rises, plasma ionizes which favors the ion-electron coupling up to a maximum value. If the temperature further increases, kinetic energy clearly overcomes the potential contribution, and coupling between particles drops. In the moderate-coupling range, interactions among particles in fact play a non-negligible role and MD calculations are therefore meaningful. For the sake of completeness, we checked that our simulation technique is robust enough to deal with strong-coupling conditions, and a good agreement between simulation and model was also found for coupling parameter values up to $\Gamma_{\text {expt. }} \approx 1$. Above this limit classical picture becomes questionable since the characteristic atomic size may become comparable to the average electron distance. Also, as expected, the results from MD simulations reproduce those from IPA simulations for the case of weakly coupled plasmas, with both comparing well with the statistical model.

At equilibrium, total energy behavior as a function of temperature is easy to interpret (see Fig. 13). At low temperatures, the system mainly consists of neutral atoms, which have a total of six degrees of freedom, i.e., three translational degrees plus three internal degrees, and therefore the total energy goes as $3 k T$. In the high-temperature limit, plasma is fully ionized; it behaves like a monoatomic ideal gas and the total energy per particle equals the translational kinetic energy, i.e., $\frac{3}{2} k T$. In the intermediate regime we observe the phase transition between neutral and fully ionized plasma states. In this region, the total energy per particle strongly increases with temperature. In other words, the required total energy to 


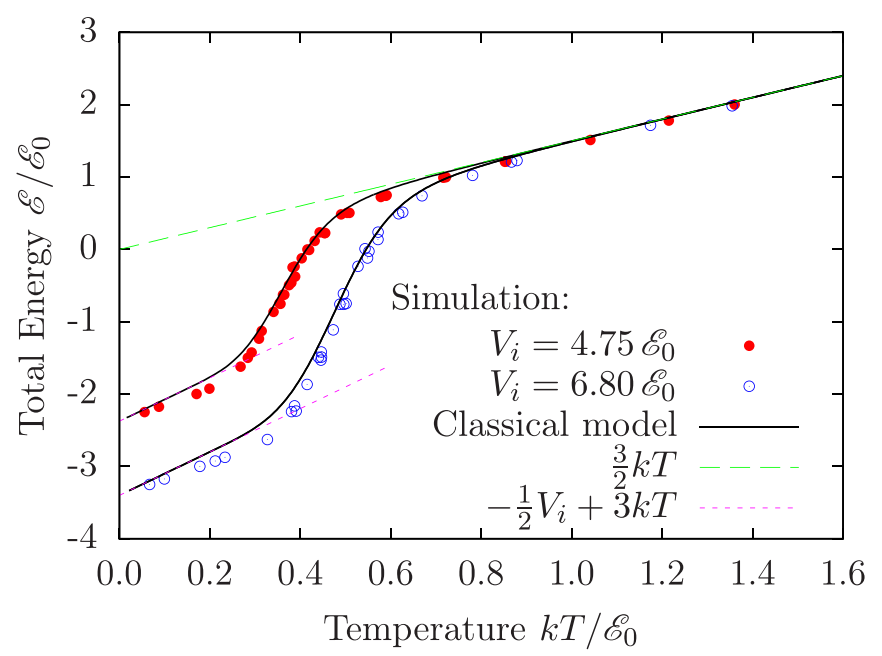

FIG. 13. Total energy per particle as a function of temperature at equilibrium. Comparisons between the analytical model and simulations are shown for two cases, i.e., $V_{i}=4.75 \mathcal{E}_{0}$ and $V_{i}=6.80 \mathcal{E}_{0}$ (with $\Gamma_{E}=0.116$ ).

produce a temperature increase is significant, because most of it is employed to overcome the neutral atom binding energy.

Further illustration of this phase equilibrium is shown in Fig. 14, where we plot the statistical distribution of potential energy per particle at particular equilibrium conditions. In the example, the plasma ionization results are $\sim 50 \%$, so that the potential energy distribution shows two well-separated bell-shaped peaks having similar area values and arising from the bound- (left one) and free- (right one) electron ensembles. Moreover, it is seen that the average potential energy of free electrons lies slightly below zero. This plasma collective effect naturally emerges from the simulation and reflects the fact that many electrons in the plasma have a negative total energy and are not bound to a particular positive ion but to the plasma as a whole, hence they sometimes are referred to as collectivized electrons [57-59]. This behavior has to do with

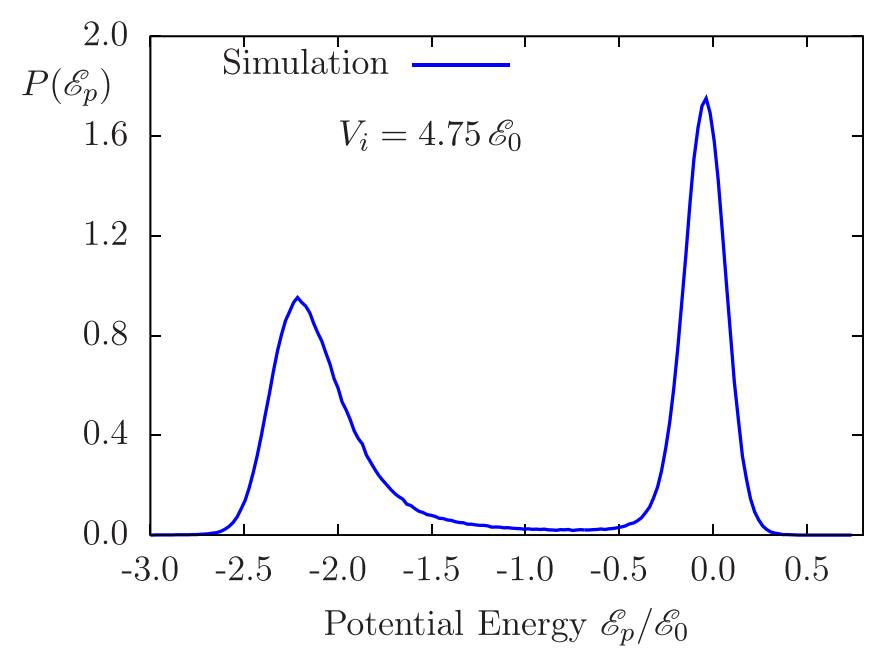

FIG. 14. Statistical distribution of electron potential energy (per particle) obtained from a simulation at equilibrium. For the case shown, equilibrium conditions are $k T=0.37 \mathcal{E}_{0}$ and $\alpha=0.53$. the effect of ionization potential depression (IPD) [57,60] that appears in dense plasmas and also illustrates how diverse the information provided by MD simulations can be. In fact, some IPD investigations in aluminum plasmas at and out of thermodynamic equilibrium have already been performed by using multicomponent classical MD simulations [61,62].

Overall, the results shown in this section reveal the robustness of the numerical algorithms implemented in the code, confirm a proper description of the ionization-recombination mechanism, and ultimately provide confidence in the physics that can be extracted from our MD simulations. Once this simulation technique has been validated, the analytical statistical model now becomes a useful tool to (a) anticipate the simulation equilibrium state and thus securely interrupt a simulation run and (b) optimize the calculation by consistently improving the choice of initial configuration when launching a simulation.

\section{CONCLUSIONS}

Particle dynamics simulations of hydrogen plasmas have been performed in the context of classical molecular dynamics. The theoretical basis of the simulation model as well as numerically relevant aspects are discussed in detail, thus proving a thorough implementation of the computer simulation technique. Particle dynamics equations are solved without using any thermostat algorithm and the simulation model properly deals with the ionization-recombination mechanism. A comprehensive study of the equilibration process is made, with an emphasis on the need for reaching the stationary stage for a safe statistical sampling of relevant physical quantities. Molecular dynamics simulations are often considered as idealized experiments, where different effects can be artificially switched on and off to assess their potential impact, thus providing deep insight into the underlying physics and a unique testbed for theory validation. However, these simulations are certainly challenging and consequently a validation process is also demanded. Here we developed an analytical statistical equilibrium model for computational validity assessment of plasma particle dynamics simulations. Good agreement between the model and molecular dynamics results was obtained in a wide range of plasma coupling parameters, thereby revealing the robustness of the employed numerical algorithms and ultimately providing confidence in the physics that can be inferred from simulation results. Continuing with the research on plasma Stark broadening performed by our group over the last three decades, the internal consistency and validity tests of MD simulations performed in this work are a first step toward the ultimate goal of carrying out a detailed investigation of the impact of ionization-recombination dynamics on broadening mechanisms of spectral line shapes from emitting ions in multicomponent plasmas (i.e., beyond a fully ionized scenario). This topic will be addressed in a forthcoming publication.

\section{ACKNOWLEDGMENTS}

This work has been supported by Research Grants No. ENE2013-42581-R/FTN and No. ENE2015-67581-R/FTN (MINECO/FEDER-UE) from the Spanish Ministry of Economy and Competitiveness. This work has been carried out 
within the framework of the EUROfusion Consortium and has received funding from the Euratom research and training programs 2014-2018 under Grant Agreement No. 633053 (Projects AWP17-ENR-IFE-CEA-01 and AWP17-ENR-IFECEA-02). The views and opinions expressed herein do not necessarily reflect those of the European Commission.

\section{APPENDIX: SOME CONSIDERATIONS ON NUMERICAL HEATING}

It is well known that combined and accumulated effect of computational errors in molecular dynamics leads to the socalled numerical heating [51-54]. In fact, the effect manifests as a total energy increase since, as can easily be demonstrated, such heating does not always result in a temperature rise. Here, we will discuss this phenomenon in order to assess both potential imposed limitations on molecular dynamics and its practical consequences when using simulation techniques for the statistical analysis of a local electric microfield.

At the time instant $t$, the total energy of a system configuration is given by

$$
\mathcal{E}=\sum_{i} \frac{1}{2} m_{i} v_{i}^{2}+\frac{1}{2} \sum_{\substack{i, j \\ i \neq j}} V\left(r_{i j}\right),
$$

with $\mathbf{r}_{i j}=\mathbf{r}_{i}-\mathbf{r}_{j}$. After each time step $\Delta t, \mathbf{v}_{i}$ and $\mathbf{r}_{i}$ will change, thus producing the consequent energy variation. Taking into account that, numerically, $\mathbf{v}(t+\Delta t)=\mathbf{v}(t)+$ $\frac{1}{m} \mathbf{F} \Delta t$, then we have

$$
\begin{aligned}
\Delta v_{i}^{2} & =v_{i}^{2}(t+\Delta t)-v_{i}^{2}(t) \\
& =2 \frac{1}{m_{i}} \mathbf{v}_{i} \cdot \mathbf{F}_{i} \Delta t+\frac{1}{m_{i}^{2}} F_{i}^{2} \Delta t^{2}, \\
\Delta V\left(r_{i j}\right) & \approx\left(\frac{\partial V}{\partial \mathbf{r}_{i j}}\right) \cdot \Delta \mathbf{r}_{i j} \\
& =-\mathbf{F}_{i j} \cdot \mathbf{v}_{i j} \Delta t \\
& =-\mathbf{F}_{i j} \cdot\left(\mathbf{v}_{i}-\mathbf{v}_{j}\right) \Delta t,
\end{aligned}
$$

so it follows

$$
\begin{aligned}
\Delta \mathcal{E} \approx & \sum_{i} \mathbf{v}_{i} \cdot \mathbf{F}_{i} \Delta t+\frac{1}{2} \sum_{i} \frac{1}{m_{i}} F_{i}^{2} \Delta t^{2} \\
& -\frac{1}{2} \sum_{\substack{i, j \\
i \neq j}} \mathbf{F}_{i j} \cdot\left(\mathbf{v}_{i}-\mathbf{v}_{j}\right) \Delta t
\end{aligned}
$$

Also,

$$
\mathbf{F}_{i}=\sum_{\substack{j \\ j \neq i}} \mathbf{F}_{i j}, \quad \text { with } \quad \mathbf{F}_{i j}=-\mathbf{F}_{j i} .
$$

Then, the first and third addends in Eq. (A3) cancel out and it is found

$$
\Delta \mathcal{E} \approx \sum_{i} \frac{1}{m_{i}} F_{i}^{2} \Delta t^{2}
$$

which is a positive quantity.

Therefore, it is said that the system numerically heats up, but it does not necessarily mean that the kinetic and potential energy separately increase. It will depend on the

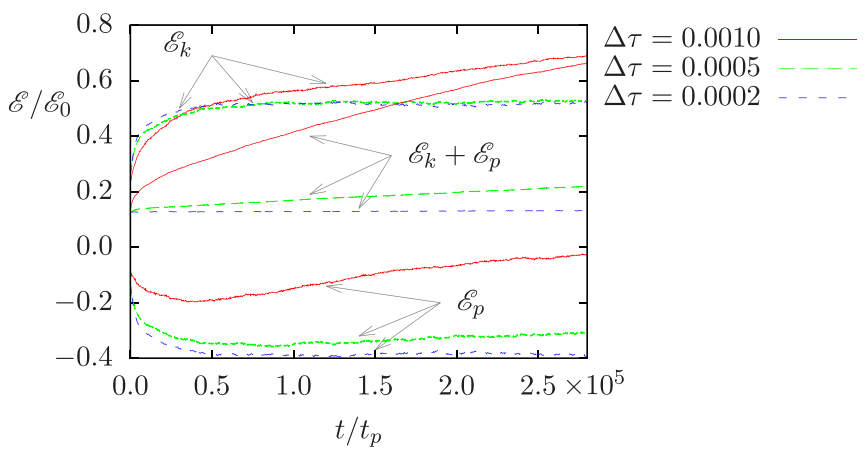

FIG. 15. Time histories of kinetic, potential, and total energy for three simulation cases. In order to assess the impact of numerical heating, different time step values, $\Delta t$, were used in each case to solve the system of motion equations.

system configuration, i.e., depending on particles location, either kinetic or potential energy could even decrease, but their sum will always increase.

We illustrate this fact in Fig. 15. The time evolution of kinetic, potential, and total energy are shown for three simulation cases (hereafter, it should be noted that each case actually represents an average over eight independent simulation runs) that were executed using different time step values. The three cases started from exactly the same initial configuration. When looking at the figure it might seem simulations start at different points, but this is because the typical sudden initial change happens in a different way for each case (note that simulation early times have not been zoomed in the figure). For the greatest time step value, numerical heating is certainly noticeable, whereas for the smallest one it is negligible. Also, under certain conditions, it is observed that numerical heating leads to an increase of potential energy per particle, while temperature remains constant -(see the case for $\Delta t=5 \times 10^{-4} t_{p}$ ).

The impact of numerical heating can be more deeply analyzed by means of Fig. 16, where trajectories in the

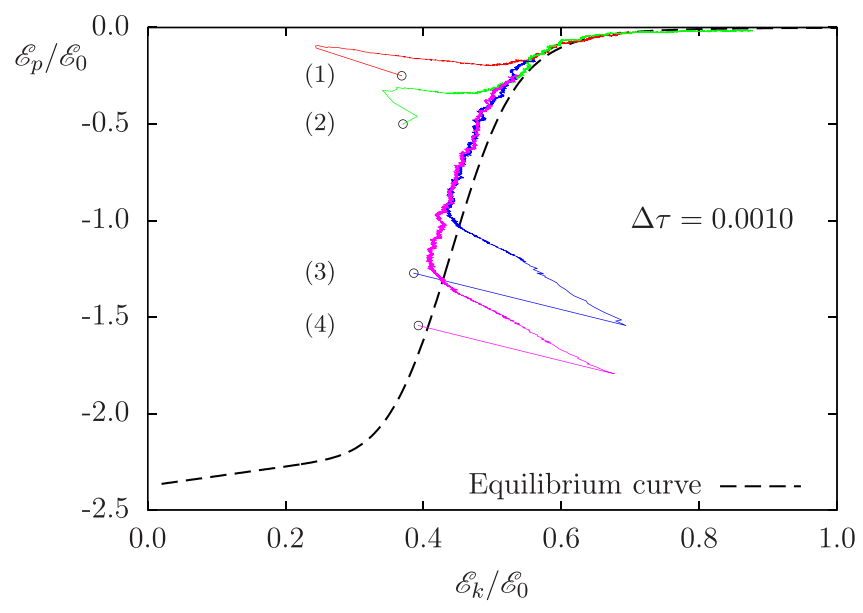

FIG. 16. Simulation trajectories in the $\mathcal{E}_{p}: \mathcal{E}_{k}$ plane. Four simulation cases launched from different initial configurations are shown. Pointedly, a coarse time step was chosen, so that numerical heating cannot be avoided and total energy increases with time in each case. 
$\mathcal{E}_{p}: \mathcal{E}_{k}$ plane are shown for four simulation cases launched from different initial conditions. In all of them the same relatively coarse time step was employed. As already mentioned, early in time a sudden exchange between kinetic and potential energy takes place. Then, the system approaches to equilibrium and the total energy continuously increases, i.e., unlike that observed in Fig. 10, and as a consequence of numerical heating, for the cases shown in Fig. 16, the path to equilibrium does not follow a $\mathcal{E}_{k}+\mathcal{E}_{p}=$ cte trajectory. In labeled cases (3) and (4), the relaxation phase develops as plasma cools down, i.e., the kinetic energy per particle decreases, so that the increase in total energy due to numerical heating actually manifests as a potential energy increase.

Once the system reaches the equilibrium curve (the one obtained from the analytical model discussed in Sec. III is displayed for reference), such total energy increase distributes among kinetic and potential energy. Still, it mainly entails an increase of potential energy, since temperature does not change too much in the process (note that different scales are used in kinetic and potential energy axes). Thus, if a thermostat algorithm, i.e., a temperature control, were used to force the simulation to stabilize, one might not realize about numerical heating because the numerically added energy amount would mostly turn into potential energy, with the kinetic one barely changing in the simulation. Nevertheless, strictly speaking, when the system begins to move following the equilibrium curve, numerical heating truly leads to a temperature increase. In fact, if the system is left to evolve for a long time it will end up in the fully ionized state. We note in passing that this picture is not consistent with a steady ionization-recombination equilibrium state.

On the other hand, since after a certain time the system evolves going through subsequent equilibrium configurations, if numerical heating slowly builds up its effect will be tolerable provided that desired information from the simulation does not require the extraction of long time histories.

Nevertheless, it should be kept in mind that the heating effect may have a greater impact on particle spatial distribution than on plasma temperature itself, so a separate surveillance of kinetic and potential energy is needed as well as a consequent handling of simulation data. In this connection, we recall that the average potential energy arises from particle spatial arrangement, which ultimately determines the microfield statistical distribution. As known, the latter plays a pivotal role in the study of Stark effect.

The analysis shown here guided the calculations performed for this work. Thus, as mentioned, in all simulation runs discussed in Sec. IV, a time step was chosen small enough to make the numerical heating effect negligible.
[1] E. Stambulchik and Y. Maron, High Energy Density Phys. 6, 9 (2010).

[2] R. Stamm and D. Voslamber, J. Quant. Spectrosc. Radiat. Transfer 22, 599 (1979).

[3] R. Stamm, B. Talin, E. L. Pollock, and C. A. Iglesias, Phys. Rev. A 34, 4144 (1986).

[4] A. Calisti, A. V. Demura, M. A. Gigosos, D. González-Herrero, C. A. Iglesias, V. S. Lisitsa, and E. Stambulchik, Atoms 2, 259 (2014).

[5] S. Ferri, A. Calisti, C. Mossé, J. Rosato, B. Talin, S. Alexiou, M. A. Gigosos, M. A. González, D. González-Herrero, N. Lara, T. Gomez, C. A. Iglesias, S. Lorenzen, R. C. Mancini, and E. Stambulchik, Atoms 2, 299 (2014).

[6] T. Nagayama, J. E. Bailey, R. C. Mancini, C. A. Iglesias, S. B. Hansen, C. Blancard, H. K. Chung, J. Colgan, P. Cosse, G. Faussurier, R. Florido, C. J. Fontes, F. Gilleron, I. E. Golovkin, D. P. Kilcrease, G. Loisel, J. J. MacFarlane, J.-C. Pain, G. A. Rochau, M. E. Sherill, and R. W. Lee, High Energy Density Phys. 20, 17 (2016).

[7] E. Stambulchik, High Energy Density Phys. 9, 528 (2013).

[8] E. Stambulchik, A. Calisti, H.-K. Chung, and M. A. González, Atoms 2, 378 (2014).

[9] J. Rosato, High Energy Density Phys. 22, 60 (2017).

[10] M. A. Gigosos and V. Cardeñoso, J. Phys. B 20, 6005 (1987).

[11] G. C. Hegerfeldt and V. Kesting, Phys. Rev. A 37, 1488 (1988).

[12] V. Cardeñoso and M. A. Gigosos, Phys. Rev. A 39, 5258 (1989).

[13] M. A. Gigosos and V. Cardeñoso, J. Phys. B 29, 4795 (1996).

[14] E. Stambulchik and Y. Maron, J. Quant. Spectrosc. Radiat. Transfer 99, 730 (2006).

[15] E. Stambulchik, S. Alexiou, H. R. Griem, and P. C. Kepple, Phys. Rev. E 75, 016401 (2007).
[16] M. A. Gigosos, J. Phys. D: Appl. Phys. 47, 343001 (2014).

[17] J. P. Hansen and I. R. McDonald, Phys. Rev. Lett. 41, 1379 (1978).

[18] J. P. Hansen and I. R. McDonald, Phys. Rev. A 23, 2041 (1981).

[19] J. P. Hansen and I. R. McDonald, Phys. Lett. A 97, 42 (1983).

[20] D. V. Fisher and Y. Maron, Eur. Phys. J. D 14, 349 (2001).

[21] H. B. Nersisyan, C. Toepffer, and G. Zwicknagel, Phys. Rev. E 72, 036403 (2005).

[22] S. P. Sadykova, W. Ebeling, I. M. Sokolov, and I. A. Valuev, Plasma Phys. Rep. 36, 1161 (2010).

[23] A. Calisti, S. Ferri, C. Mossé, B. Talin, M. A. Gigosos, and M. A. González, High Energy Density Phys. 7, 197 (2011).

[24] S. Hau-Riege and J. Weisheit, Phys. Rev. E 91, 033106 (2015).

[25] S. P. Hau-Riege and J. Weisheit, Phys. Rev. E 95, 013204 (2017).

[26] S. Ferri, A. Calisti, C. Mossé, B. Talin, M. A. Gigosos, and M. A. González, High Energy Density Phys. 3, 81 (2007).

[27] E. Stambulchik, D. V. Fisher, Y. Maron, H. R. Griem, and S. Alexiou, High Energy Density Phys. 3, 272 (2007).

[28] H. B. Nersisyan, C. Toepffer, and G. Zwicknagel, Contrib. Plasma Phys. 50, 193 (2010).

[29] B. Talin, A. Calisti, E. Dufour, and J. Dufty, J. Quant. Spectrosc. Radiat. Transfer 71, 729 (2001).

[30] B. Talin, A. Calisti, and J. Dufty, Phys. Rev. E 65, 056406 (2002).

[31] A. Calisti, T. del Río Gaztelurrutia, and B. Talin, High Energy Density Phys. 3, 52 (2007).

[32] F. R. Graziani, V. S. Batista, L. X. Benedict, J. I. Castor, H. Chen, S. N. Chen, C. A. Fichtl, J. N. Glosli, P. E. Grabowski, A. T. Graf, S. P. Hau-Riege, A. U. Hazi, S. A. Khairallah, L. Krauss, A. B. Langdon, R. A. London, A. Markmann, M. S. 
Murillo, D. F. Richards, H. A. Scott, R. Shepherd, L. G. Stanton, F. H. Streitz, M. P. Surh, J. C. Weisheit, and H. D. Whitley, High Energy Density Phys. 8, 105 (2011).

[33] A. Calisti, S. Ferri, and B. Talin, High Energy Density Phys. 5, 307 (2009).

[34] A. Calisti and B. Talin, Contrib. Plasma Phys. 51, 524 (2011).

[35] S. P. Hau-Riege, J. Weisheit, J. I. Castor, R. A. London, H. A. Scott, and D. F. Richards, New J. Phys. 15, 015011 (2013).

[36] N. Lara, Calculations of Stark spectra of strongly coupled plasmas by molecular dynamics simulation, Ph.D. thesis, University of Valladolid, Spain, 2013.

[37] D. González-Herrero, Study of the Stark broadening of the $3 s-3 p$ spectral lines in Be-like ions-Molecular dynamics simulations, Ph.D. thesis, University of Valladolid, Spain, 2016.

[38] G. Kelbg, Ann. Phys. 467, 219 (1963).

[39] T. Dunn and A. A. Broyles, Phys. Rev. 157, 156 (1967).

[40] C. Deutsch, Phys. Lett. A 60, 317 (1977).

[41] G. E. Norman and A. A. Valuev, Plasma Phys. 21, 531 (1979).

[42] H. Minoo, M. M. Gombert, and C. Deutsch, Phys. Rev. A 23, 924 (1981).

[43] W. Ebeling, G. E. Norman, A. A. Valuev, and I. A. Valuev, Contrib. Plasma Phys. 39, 61 (1999).

[44] A. V. Filinov, M. Bonitz, and W. Ebeling, J. Phys. A 36, 5957 (2003).
[45] W. Ebeling, A. Filinov, M. Bonitz, V. Filinov, and T. Pohl, J. Phys. A 39, 4309 (2006).

[46] M. Y. Amusia and L. V. Chernysheva, Computation of Atomic Processes: A Handbook for the ATOM Programs (Institute of Physics Publishing, Bristol, 1997).

[47] L. V. Chernysheva and V. L. Yakhontov, Comput. Phys. Commun. 119, 232 (1999).

[48] M. F. Gu, Can. J. Phys. 86, 675 (2008).

[49] L. Verlet, Phys. Rev. 159, 98 (1967).

[50] L. Verlet, Phys. Rev. 165, 201 (1968).

[51] R. W. Hockney and J. W. Eastwood, Computer Simulation Using Particles (Taylor \& Francis, London, 1988).

[52] H. Ueda, Y. Omura, H. Matsumoto, and T. Okuzawa, Comput. Phys. Commun. 79, 249 (1994).

[53] P. W. Rambo, J. Comput. Phys. 133, 173 (1997).

[54] E. G. Evstatiev and B. A. Shadwick, J. Comput. Phys. 245, 376 (2013).

[55] P. H. Hünenberger, Adv. Polym. Sci. 173, 105 (2005).

[56] M. N. Saha, Philos. Mag. 40, 472 (1920).

[57] D. V. Fisher and Y. Maron, Eur. Phys. J. D 18, 93 (2002).

[58] A. V. Lankin and G. E. Norman, Dokl. Phys. 53, 51 (2008).

[59] A. V. Lankin and G. E. Norman, J. Phys. A 42, 214032 (2009).

[60] B. J. B. Crowley, High Energy Density Phys. 13, 84 (2014).

[61] A. Calisti, S. Ferri, and B. Talin, J. Phys. B 48, 224003 (2015).

[62] A. Calisti, S. Ferri, and B. Talin, Contrib. Plasma Phys. 55, 360 (2015). 